Rev. Elev, Méd. vét. Pays trop., 1972, 25 (4) : 521-525

\title{
Incidence sérologique des anticorps anti-brucelliques chez les animaux domestiques de l'homme en Iran
}

\author{
par H. TADJEBAKHCHE $(*)$ et A. GATEL $\left({ }^{*}\right)$
}

\begin{abstract}
RESUME
L'incidence des anticorps anti-brucelliques en Iran a été recherchée sur 3.647 sérums récoltés chez les principales espèces animales domestiques et chez l'homme. Les pourcentages d'infectés sont :

Ovins 4,24 p .100 (mâles 2,6 p. 100 - femelles 4,3 p. 100), Caprins 2,18 p. 100 , Bovins 12 p. 100 (veaux 8,2 p. 100 - mâles 4,7 p. 100 - femelles 16,1 p. 100), Equins 0,73 p. 100, Porcins 17,6 p. 100, Canins 4,87 p. 100 , Buffles 5,5 p. 100 , Homme 5,5 p. 100 (hommes 5 p. 100 - fermmes 6,8 p. 100).

Les résultats obtenus par séro-agglutination (S.A.L.) et fixation du complément (F.C.') ont été comparés sur 674 sérums:

- 79,5 p. 100 des sérums positifs en S.A.L. sont positifs en F.C.';

- 29,3 p. 100 des sérums suspects en S.A.L. sont positifs en F.C.';

- $\quad 9,9$ p. 100 des sérums négatifs en S.A.L. sont positifs en F.C.'

Compte tenu des pourcentages de positivité obtenus, l'établissement de mesures rigoureuses concernant la prophylaxie de cette maladie en Iran est suggéré.
\end{abstract}

La brucellose est en Iran la cause primordiale d'avortement chez les animaux domestiques et est responsable d'importantes pertes économiques, surtout dans la zone d'élevage intensif autour de Téhéran. Quelques enquêtes sont régulièrement effectuées chez les bovins et ovins, dans le cadre du dépistage de cette maladie $(9,10,11)$; mais elles restent limitées au secteur géographique de Téhéran.

Notre propos dans ce travail a été de diversifier notre échantillonnage de sérum, tant du point de vue origine géographique que du point de vue espèce.

(*) Université de Téhéran. Faculté de Médecine Vétérinaire. Chaire de Microbiologie et Maladies contagieuses, B.P. na 3262, Téhéran-Iran.

(**) Coopérant technique, appelé du Service national.

\section{MATERIEL ET METHODES}

\section{a) Origine des sérums}

Les sérums de bovins, d'ovins, de caprins et de buffles ont été récoltés à l'ábattoir de Téhéran sur des animaux de provenances diverses (Azerbaidjan, Khorasan, Mazandéran, Baluchistan, Téhéran... etc.).

Les sérums de porcins proviennent de l'abattoir "Arzouman " de Téhéran.

Les sérums d'équins proviennent soit de la chaire d'anatomie et d'histologie de la Faculté Vétérinaire de Téhéran, soit de l'Institut Razi.

Les sérums canins proviennent des cliniques de la Société protectrice des animaux de Téhéran.

Les sérums humains ont été obtenus auprès de patients, dans des laboratoires d'analyses médicales. Les prélèvements chez ces patients 
étaient effectués pour d'autres motifs qu'une éventuelle atteinte brucellique.

\section{b) Séro-agglutination (S.A.L.)}

Nous avons retenu une technique de séroagglutination lente en tube $(1,5,7,13)$, les dilutions allant de $1 / 10$ à $1 / 5120$. Pour limiter les phénomènes de zones, nous avons dilué les sérums avec une solution aqueuse de $\mathrm{NaCl}$ à 5 p. 100.

L'antigène a été fourni par l'Institut Razi. Il est préparé à partir de la souche Brucella abortus 99 et est agglutiné à ++ au $1 / 500$ par le sérum étalon international (titrant luimême 1.000 U.I.) (2).

Les seuils de positivité suivants ont été retenus :

- 100 U.I. (3/40) pour les sérums de bovins, de porcins, de canins, d'équins, de buffles et d'hommes $(1,3,4,8,13)$.

- 60 U.I. $(3 / 20)$ pour les sérums ovins et caprins $(1,5,7)$.

Ont été considérés comme suspects les sérums titrant entre :

- 30 U.I. et 100 U.I. pour les bovins, les équins, les buffles.

- 25 U.I. et 60 U.I. pour les ovins et les caprins.

- 50 U.I. et 100 U.I. pour les porcins.
Pour l'homme et le chien, ont été considérés comme suspects les sérums présentant une agglutination comprise entre 20 U.I. $(2 / 10)$ et 100 U.I. $(3 / 40)$.

\section{c) Fixation du complément (F.C.')}

Nous avons utilisé une méthode de fixation à froid de type Kolmer $(1,5,7,13,14)$, les dilutions allant du $1 / 4$ au $1 / 1024$.

L'antigène est celui employé pour la S.A.L.; il est lavé trois fois au tampon véronal $(\mathrm{pH} 7,3)$ et dilué au $100^{\circ}$ pour les animaux et au $150^{\mathrm{B}}$ pour l'homme.

Tous les sérums suspects en S.A.L., ainsi que quelques positifs et négatifs, ont été soumis à la F.C.' Tous les sérums fixant le complément au 1/4 ont été considérés comme positifs (4, 13).

\section{RESULTATS}

\section{Pourcentages de positifs}

Nous avons établi nos pourcentages en considérant comme positifs les sérums positifs en S.A.L. et les sérums suspects en S.A.L. et positifs en F.C.'

Le tableau I donne le nombre de sérums examinés et le pourcentage de positifs.

TABLEAU $\mathrm{N}^{*}$ I

\begin{tabular}{|c|c|c|c|c|c|c|}
\hline & $\begin{array}{l}\text { Nombre de } \\
\text { sêrums }\end{array}$ & $\begin{array}{l}\text { S.A.L. } \\
\text { positives }\end{array}$ & $\begin{array}{l}\text { S.A.L. } \\
\text { suspectes }\end{array}$ & $\begin{array}{l}\text { S.A.L. suspectes } \\
\text { à F.C' positives }\end{array}$ & \begin{tabular}{|} 
S.A.L. positives + \\
S.A.L. suspectes \\
a F.C' positive
\end{tabular} & Pourcentage \\
\hline Ovins & 1.021 & 31 & 96 & 20 & 51 & 4,24 \\
\hline Caprins & 640 & 9 & 37 & 5 & 14 & 2,18 \\
\hline Bovins & 923 & 80 & 111 & 31 & 111 & 12,0 \\
\hline Equins & 273 & 1 & 24 & 1 & 2 & 0,73 \\
\hline Porcins & 227 & 27 & 36 & 13 & 40 & 17,6 \\
\hline Canins & 41 & 1 & 1 & 1 & 2 & 4,87 \\
\hline Buffle & 36 & 1 & 4 & 1 & 2 & 5,5 \\
\hline Homme & 306 & 12 & 36 & 6 & 18 & 5,5 \\
\hline Total & 3.467 & 162 & 345 & 78 & 240 & \\
\hline
\end{tabular}

Dans le tableau II sont distingués, d'une part le sexe et l'âge des bovins, d'autre part le sexe pour les sérums humains et ovins (il est à noter que cette étude n'a pu être possible que sur 757 des sérums ovins recueillis). 
TABLEAU N*II

\begin{tabular}{|lc|c|c|c|}
\hline & & $\begin{array}{c}\text { Nombre de } \\
\text { sérums }\end{array}$ & Positifs & Pourcentage \\
\hline Homme & M & 160 & 8 & 5,0 \\
\multirow{4}{*}{ Ovins } & F & 146 & 10 & 6,84 \\
& M & 115 & 3 & 2,6 \\
Bovins-Veaux & 182 & 15 & 8,36 \\
& F & 642 & 28 & 8,24 \\
& M & 210 & 10 & 4,7 \\
& F & 531 & 86 & 16,1 \\
\hline
\end{tabular}

II. Comparaison entre S.A.L. et F.C.'

Nous avons effectué le test de F.C.' sur 674 sérums des 3.647 déjà examinés en S.A.L.

Les résultats sont consignés dans le tableau III.

TABLEAU $N^{\circ}$ III

\begin{tabular}{|c|c|c|c|c|c|c|c|}
\hline & $\begin{array}{c}\text { S.A.L. } \\
\text { positive } \\
\text { F.C' } \\
\text { positive }\end{array}$ & $\begin{array}{l}\text { S.A.L. } \\
\text { positive } \\
\text { F.C' } \\
\text { nëgative }\end{array}$ & $\begin{array}{l}\text { S.A.L. } \\
\text { suspecte } \\
\text { F.C' } \\
\text { positive }\end{array}$ & $\begin{array}{c}\text { S.A.L. } \\
\text { suspecte } \\
\text { F.C' } \\
\text { négative }\end{array}$ & $\begin{array}{c}\text { S.A.L. } \\
\text { négative } \\
\text { F.C' } \\
\text { positive }\end{array}$ & $\begin{array}{c}\text { S.A.L. } \\
\text { négative } \\
\text { F.C' } \\
\text { négative }\end{array}$ & $\begin{array}{c}\text { Nombre } \\
\text { de } \\
\text { sêrums }\end{array}$ \\
\hline Bovins & 29 & & 31 & 46 & 5 & 37 & 148 \\
\hline Ovins & 16 & 7 & 20 & 64 & 9 & 57 & 173 \\
\hline Caprins & 5 & 4 & 5 & 32 & 6 & 55 & 107 \\
\hline Equins & 1 & & 1 & 14 & 3 & 15 & 34 \\
\hline Porcins & 14 & 5 & 13 & 23 & 4 & 13 & 72 \\
\hline Canins & 1 & & 1 & & & 6 & 8 \\
\hline Bufrles & 1 & & 3 & & & & 4 \\
\hline Homme & 3 & 2 & 6 & 14 & 4 & 99 & 128 \\
\hline ] l'ot & 70 & 18 & 80 & 193 & 31 & 282 & 674 \\
\hline
\end{tabular}

\section{DISCUSSION}

Selon les rapports de l'Institut Razi et des Services Vétérinaires d'Iran, le pourcentage d'infectés est de l'ordre de 20 p. 100 chez les bovins de la zone d'élevage intensif de Téhéran et de 15 p. 100 chez les ovins des zones d'élevage de Téhéran et d'Isfahan $(9,10,11)$. Les résultats que nous avons obtenus sont différents $(12$ p. 100 chez les bovins et 4,2 p. 100 chez les ovins du fait que notre échantillonnage provient en majeure partie de zones à élevage extensif et d'aires géographiques plus diverses.

Chez les espèces bovines et ovines pour lesquelles nous avons pu distinguer le sexe, les femelles sont plus infectées que les mâles (tableau II) tandis que chez l'homme aucune différence notable n'apparaît.

Sur 674 sérums d'espèces différentes (des 3.647 sérums étudiés en S.A.L.) soumis aux deux tests S.A.L. et F.C.' (tableau III),
79,5 p. 100 des 88 sérums positifs en S.A.L. ont une F.C.' positive et 20,5 p. 100 une F.C.' négative. Il est à noter que ces derniers sont à la limite de positivité en S.A.L.

Sur 273 sérums suspects en S.A.L., 29,3 p. 100 ont une F.C.' positive.

Sur 313 sérums négatifs en S.A.L., 9,9 p. 100 ont une F.C.' positive.

Cela confirme la plus grande sensibilité de la méthode de F.C.' par rapport à la S.A.L. $(2,4,5,6,14)$ et montre la concondance et la non-concordance des résultats en S.A.L. et F.C.' dans le diagnostic de la brucellose.

\section{CONCLUSIONS}

La brucellose sévit chez toutes les espèces animales et l'homme en Iran. Il ne faut pas négliger l'importance des espèces porcines, équines, canines dans l'épizootiologie et l'épidémiologie de cette maladie. 
Pour le diagnostic sérologique, il est recommandé d'effectuer les deux tests (S.A.L., F.C.') simultanément. Eventuellement, dans le cadre d'une enquête sérologique portant sur un nombre important de prélèvements, on pourrait se contenter de faire une F.C.' sur tous les suspects en S.A.L.

Les pourcentages de positivité obtenus suggèrent la nécessité de la mise en place en Iran de mesures plus rigoureuses concernant le dépistage et la prophylaxie de cette maladie.

\section{Remerciements}

Nous tenons à remercier vivement : MM. les Professeurs Rafyi, Chimie pour les facilités qu'ils nous ont accordées dans l'élaboration de ce travail. M. le Dr Ebadi de l'Institut Razi qui a bien voulu nous fournir l'antigène. M. Attei qui nous a aimablement procuré des sérums humains ainsi que M. le Dr Farges pour son excellente assistance et M. le Dr Keyvanfar.

\section{SUMMARY}

\section{Serological incidence of Brucella-antibodies in domestic animals and man in Iran}

The occurence of brucella-antibody among the different species of domestic animals in Iran has been studied. The percentage of infected animals are as follow: sheep 4,24 p. 100 (female 4,3 p. 100 - male 2,6 p. 100 ), goats 2,18 p. 100 , cattle 12 p. 100 (calves 8,2 p. 100 - male 4,7 p. 100 - female 16,1 p. 100 ), horses 0,73 p. 100 , swines 17,6 p. 100 , dogs 4,87 p. 100 , buffaloes 5,5 p. 100 , man 5,5 p. 100 (masc. 5 p. 100 , fem. 6,8 p. 100).

The results of two S.A.T. and C.F.T. has been compared :

- 79,5 p. 100 of serums positive by S.A.T. were also positive by C.F.T.

$-29,3$ p. 100 of suspicious serums by S.A.T. were positive by C.F.T.

- 9,9 p. 100 of negative serums by S.A.T. were positive by C.F.T.

Because of the distribution of the disease in the different species of animals strict control procedures are essential.

\section{RESUMEN}

Incidencia serológica de los anticuerpos contra la brucelosis en los animales domésticos y el hombre en Iran

Se buscó la incidencia de los anticuerpos contra la brucelosis en Iran en 3.647 sueros recogidos en las principales especies animales domésticas $y$ en el hombre.

Los porcentajes de animales infectados son los siguientes: oveja: 4,24 p. 100 (machos 2,6 p. 100 ; hembras 4,3 p. 100), cabra 2,18 p. 100 , bovinos 12 p. 100 (terneros 8,2 p. 100 - machos 4,7 p. 100 ; hembras 16,1 p. 100), equinos 0,73 p. 100 , porcinos 17,6 p. 100 , caninos 4,87 p. 100 , búfalos 5,5 p. 100 , hombre 5,5 p. 100 (machos 5 p. 100 - hembras 6,8 p. 100).

Se compararon los resultados obtenidos mediante la sero-aglutinación (s. a.) y la fijación del complemento (f. c.) en 674 sueros.

- 79,5 p. 100 de los sueros positivos en s. a. son positivos en f.c.

- 29,3 p. 100 de los sueros sospechosos en s.a. son positivos en f. c.

- 9,9 p. 100 de los sueros negativos en s. a. son positivos en f.c.

Teniendo en cuenta los porcentajes de positividad obtenidos, se sugiere el establecimiento de medios rigurosos de profilaxis de dicha enfermedad en Iran. 


\section{BIBLIOGRAPHIE}

1. ALTON (G. G.) et JONES (L.). F.A.O. Working Document, Animal Health Branch Monograph, no 7., Rome, F.A.O., 1963.

2. EBADI (A.). Comparison of various serological tests on the milk of sheep in relation to the isolation of Brucella melitensis. Brit. vet. J., 1971, 127: 105-112.

3. EL BAHRI (L.), BEN OSMAN (F.) et CHADLI (A.). Enquête sérologique sur la brucellose canine en Tunisie. Arch. Inst. Pasteur Tunis, 1970, 43: 315-331.

4. GERAL (M. F.), LASSERE (J.), SAURAT (P.), LAUTIE (R.), CHANTAL (J.) et MEIGNIER (B.). Enquête sérologique sur l'infection brucellique des étudiants vétérinaires toulousains, Rev. Méd. vét., 1971, $122: 415-432$.

5. MORGAN (B. W. J.). The serological diagnosis of bovine brucellosis. Vet. Rec., 1967, 80: 612621.

6. OPITZ (H. M.). Brucellosis in Sierra Leone, a serological survey in cattle, sheep and goats. Bull. epizoot. Dis. Afr., 1969, 17 : 383-391.

7. PILET (Ch.), TOMA (B.) et BONNEAU (M.) Sur le choix d'une technique de fixation du com- plément adaptée au diagnostic de la brucellose. Ann. Inst. Pasteur, 1967, 113: 984-950.

8. PILET (Ch.) et TOMA (B.). Les techniques de séro-agglutination dans le diagnostic de la brucellose. Cah. Méd. vét., 1969, 38 : 3-22.

9. Rapport Annuel de l'Institut Razi, 1968, Téhéran, 544 p.

10. Rapport Annuel de l'Institut Razi, 1969, Téhéran, $449 \mathrm{p}$.

11. Rapport Annuel des Services Généraux Vétérinaires d'Iran, 1968, Téhéran, 153 p.

12. RENOUX (G.). Etude sur la brucellose ovine et caprine. XV. Du diagnostic sérologique de la brucellose individuelle de chèvres artificiellement infectées par Brucella melitensis. Arch. Inst. Pasteur Tunis, 1957, 34: 207-225.

13. RENOUX (G.) et GAUMONT (R.). Pathologie de la production du lait. II. Méthodes de diagnostic des brucelloses animales. Ann. Nutr. Alim., 1966, 20 : $1-51$.

14. WISNIOWSKI (J.). The role of the complement fixation test in the serological diagnosis of brucellosis in cattle. Brit. vet. J., 1964, 120 : 15-20. 